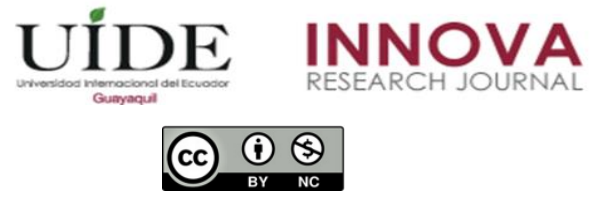

INNOVA Research Journal, ISSN 2477-9024

(Enero-Abril 2020). Vol. 5, No.1 pp. 111-128

DOI: https://doi.org/10.33890/innova.v5.n1.2020.1115

URL: http://revistas.uide.edu.ec/index.php/innova/index

Correo: innova@uide.edu.ec

\title{
Estrategias competitivas de las empresas ecuatorianas exportadoras de camarón. Casos de éxito
}

\section{Competitive strategies of the exporting ecuadorian companies exporting of shrimp. Success cases}

Grace Geanella Moncada-Sánchez

Paula Ximena Ramírez-Quevedo

Mayiya Lisbeth González-Illescas

Universidad Técnica de Machala, Ecuador

Autor para correspondencia: gmoncada_est@utmachala.edu.ec;

pxramirez_est@utmachala.edu.ec; mlgonzalez@utmachala.edu.ec

Fecha de recepción: 31 de julio del 2019 - Fecha de aceptación: 05 de noviembre del 2019

\section{Resumen}

El propósito de esta investigación es identificar los tipos de estrategia que han implementado las empresas exportadoras de camarón para mantenerse en el entorno dinámico de los mercados internacionales. La investigación se fundamentó en la comparación de las teorías y enfoques procedentes de la revisión bibliográfica. Se utilizó el método de estudio de caso, mediante un enfoque cualitativo. La muestra de estudio está compuesta por cinco empresas camaroneras seleccionadas de acuerdo al puesto que ocupan en el ranking nacional de exportadoras que cuentan con mayor participación en su volumen de ventas. Entre los principales hallazgos, se destaca que las empresas analizadas han ejecutado: estrategias de crecimiento (integración vertical y diversificación) y estrategias competitivas (diferenciación), asegurando una producción responsable y de calidad. Finalmente se hace referencia a la importancia de las alianzas entre el sector empresarial con la Cámara Nacional de Acuicultura y el Ministerio de Comercio Exterior como estrategia para aprovechar las oportunidades del mercado.

Palabras Claves: estrategia; ventaja competitiva; innovación; alianzas; sector camaronero

\begin{abstract}
The purpose of this research is to identify the types of strategies that shrimp exporting companies have implemented to be in the dynamic setting of international markets. The research is based on the comparison of theories and approaches the literature review. The research used a case study method, through a qualitative approach. Five shrimp companies participated in this study according to the position in the national ranking of exporters they have greater participation volume of sales. Among the main findings, the analyzed companies have executed growth strategies (vertical integration and diversification) and competitive strategies (differentiation), ensuring responsible and quality production. The National Camera of Aquaculture and the Minister of Foreign Trade participated as alliances as a strategy that has made it possible to take advantage of market opportunities.
\end{abstract}


Key Words: strategy; competitive advantage; innovation; alliances; shrimp sector

\section{Introducción}

La dinámica actual del mercado global exige el replanteamiento de las estrategias competitivas de las empresas para poder mantener su posicionamiento. En el diseño de las estrategias es necesario combinar el resultado del análisis de la situación interna de la entidad y su capacidad de mejora para suplir las necesidades del mercado externo (Trillo y Hernández, 2016).

La complejidad del entorno comercial internacional impulsa a las empresas a efectuar acciones de cambio. La adaptación a las transformaciones del ambiente de negocios no solo contribuye al aumento de la eficiencia y eficacia en las operaciones para aprovechar las oportunidades del mercado, al mismo tiempo se defiende la permanencia de las empresas (Gallo, 2017).

En Ecuador, las empresas del sector camaronero experimentaron en los años 90 una dura crisis sanitaria, la misma que repercutió con un amplio efecto multiplicador en la dinámica de la economía nacional. En un escenario que reflejaba condiciones adversas para los cultivos de camarón, se implementaron un conjunto de acciones que reflejaron cambios en las prácticas de producción. Se desarrollaron procesos de mejora continua a través de investigaciones asociadas a las larvas, formas de alimentación e incorporación de tecnología. La explotación de manera intensiva fue reemplazada por la explotación extensiva de baja densidad, consiguiendo así la recuperación del sector.

En efecto, el crecimiento acelerado del sector camaronero ha ocasionado la expansión de laboratorios de larvas, plantas procesadoras y exportadoras. Hasta el presente, operan 39 empresas exportadoras y 1.315 productoras de camarón. En consecuencia, el sector origina más de 200.000 plazas de empleo (Revista Líderes, 2017).

El sector camaronero ha registrado en los últimos cinco años un crecimiento sostenido (Banco Central del Ecuador, 2015). Lo ingresos generados por las exportaciones de camarón se constituyen en una importante fuente de divisas en una economía dolarizada, siendo objeto de priorización de políticas públicas. Cabe destacar que se registra un mayor número de empresas que sólo realizan operaciones del primer eslabón de la cadena productiva, es decir, existen empresas productoras de camarón que participan como proveedoras de las empresas exportadoras. El sector también se compone de empresas que son productoras y exportadoras y realizan toda la operación de la cadena productiva y de comercialización. Precisamente, sobre estas empresas se enfoca el presente estudio. En este sentido, nos motiva conocer ¿qué estrategias utilizan las empresas exportadoras de camarón para mantenerse en el contexto de cambios acelerados en los mercados?

Definida la interrogante que guía esta investigación, nos planteamos el objetivo de identificar las estrategias que han desarrollado las empresas exportadoras de camarón para ser competitivas y enfrentar la dinámica de los mercados internacionales. Bajo la premisa de que el dinamismo del mercado de productos alimenticios engendra oportunidades y amenazas para los 
actores del comercio internacional, a las empresas exportadoras de camarón se les presenta la necesidad de desarrollar estrategias para generar ventajas competitivas.

Como estructura del documento se presenta la introducción, se realiza un enlace entre estrategia y competitividad a partir de precisiones teóricas, se analiza la situación del sector camaronero ecuatoriano perfilando a las empresas con mayor participación en las exportaciones para su selección en el estudio. La metodología empleada se define como estudio de caso, para lo cual empleamos fuentes bibliográficas, información de las páginas web de las empresas, publicaciones nacionales y extranjeras. Por último, se analizan los resultados de la investigación seguido de las conclusiones, la identificación de limitaciones y las futuras líneas de investigación que consideramos pueden ser útiles para el sector.

\section{Revisión de la literatura}

\section{La estrategia en el ámbito empresarial}

Siguiendo a Tarapuez, Guzmán y Parra (2016), la estrategia empresarial ha sido explicada por diversos autores, no obstante, la diferencia de enfoques en su análisis, los autores concuerdan en que la estrategia es un mecanismo sustancial para el éxito de las empresas. Las organizaciones que anhelan alcanzar el éxito consideran a la estrategia como la herramienta base para impulsar el desarrollo, el rendimiento y la competitividad.

La estrategia analizada desde varias perspectivas tiene múltiples significados. Entre los autores cuyos planteamientos han tenido gran acogida en el ámbito académico, se identifica a Chandler (1962), quien se refiere a la estrategia como la ejecución de objetivos a través de la asignación de recursos a largo plazo. Por otro lado, Andrews (1971), destaca que la posición de un negocio está a cargo de la estrategia cuya planificación se compone de objetivos, políticas, planes, metas y propósitos de una organización.

En el ámbito de los negocios, la estrategia se encuentra estrechamente vinculada con la capacidad que tiene una empresa para enfrentar al dinamismo y satisfacer las imposiciones del mercado. De acuerdo a Ohmae (1989), la estrategia es el medio por el cual la organización emplea sus fortalezas para crear un factor diferenciador frente a sus competidores, sometiéndose a los requerimientos del consumidor final y el entorno en que se desarrolla.

Thompson (1994), señala que la estrategia se fundamenta en la planificación que una empresa diseña y desarrolla para cumplir sus metas propuestas, en tanto Ansoff (1998), lo interpreta como un patrón que enmarca las directrices necesarias que la empresa debe seguir.

Jones y George (2006), definen a la estrategia como la mezcla de decisiones que abarcan todas las actividades para explotar recursos, cuyo fin está en el logro de las metas de una organización. Para recoger la visión contemporánea de otros autores, en la tabla 1 se expone la concepción de estrategia. 
Tabla 1.

Definiciones de Estrategia.

\begin{tabular}{cl}
\hline Autor & \multicolumn{1}{c}{ Descripción } \\
\hline \multirow{2}{*}{ Ortíz y Arredondo, (2014) } & $\begin{array}{l}\text { La estrategia es un conjunto de capacidades que conllevan al éxito empresarial, } \\
\text { contribuyendo satisfactoriamente a la administración de recursos y a adaptarse a } \\
\text { los cambios del entorno. }\end{array}$ \\
\hline \multirow{2}{*}{$\begin{array}{c}\text { Mora-Riapira, Vera-Colina y } \\
\text { Melgarejo-Molina, (2015) }\end{array}$} & $\begin{array}{l}\text { La estrategia empresarial basada en la visión del negocio es un componente } \\
\text { imprescindible para lograr una postura fundamental en el mercado objetivo. }\end{array}$ \\
\cline { 2 - 3 } & $\begin{array}{l}\text { La estrategia está direccionada en fortalecer la planificación e incrementar la } \\
\text { competitividad, debido a que se constituye el eje central para el desarrollo de } \\
\text { actividades. }\end{array}$ \\
\hline Ahumada y Perusquia, (2016) & $\begin{array}{l}\text { Las prácticas innovadoras que involucran a los procesos productivos, al } \\
\text { mejoramiento del recurso tecnológico, y los cambios en la cultura organizacional } \\
\text { son estrategias que permiten el desarrollo empresarial. }\end{array}$ \\
\hline Tarapuez, Guzmán y Parra, & $\begin{array}{l}\text { La estrategia es el medio en que una empresa se vale de sus fortalezas para } \\
\text { conseguir ventaja frente a la competencia, sin dejar de lado los requerimientos } \\
\text { del cliente y el entorno. }\end{array}$ \\
\hline Manfredi y González, (2017) & $\begin{array}{l}\text { La estrategia es un recurso indispensable para la solución de problemas y } \\
\text { obtención de resultados óptimos en las negociaciones. }\end{array}$ \\
\hline Capa, Benítez y Capa, (2018) & $\begin{array}{l}\text { La estrategia se elabora para aprovechar las oportunidades de crecimiento de la } \\
\text { entidad en el mercado. La efectividad en sus resultados está vinculada con la } \\
\text { planificación y control de los líderes. }\end{array}$ \\
\hline
\end{tabular}
Fuente: Elaboración propia.

A partir de la revisión de las definiciones propuestas por los autores citados, consideramos que la estrategia se constituye en un mecanismo para alcanzar los objetivos de la empresa, mediante planes de acción coherentes con los cambios del entorno y la capacidad organizacional, a fin de conseguir las adaptaciones necesarias a las exigencias en curso.

En la revisión de literatura se identifican tipos de estrategias que se diferencian por sus orientaciones específicas, con las cuales contribuyen a perfilar el camino que la empresa debe seguir para cumplir los objetivos estratégicos. En la tabla 2 se presenta los tipos de estrategias de mayor difusión y aceptación en el ambiente académico y empresarial. 
Tabla 2.

Tipos de Estrategia.

\begin{tabular}{|c|c|c|}
\hline Autor & Tipo de Estrategia & Conceptualización \\
\hline Baena, (2011) & Alta segmentación & $\begin{array}{l}\text { Analiza en un conjunto de consumidores las necesidades } \\
\text { semejantes y el comportamiento de compra, dando la } \\
\text { oportunidad a que la empresa cree un producto y/o servicio } \\
\text { específico, con el objetivo de saciar las necesidades del } \\
\text { conjunto de consumidores de manera rentable para la entidad. }\end{array}$ \\
\hline Luna, (2014) & Liderazgo en costos & $\begin{array}{l}\text { Tiene como objetivo disminuir los costos en todo el proceso } \\
\text { productivo sin afectar la calidad del bien, aprovechando la } \\
\text { disponibilidad de sus recursos para obtener una ventaja } \\
\text { competitiva. }\end{array}$ \\
\hline $\begin{array}{l}\text { Thompson et al., } \\
\text { (2015) }\end{array}$ & Integración vertical & $\begin{array}{l}\text { Consiste en expandir las operaciones de la empresa, } \\
\text { involucrando a las fases de aprovisionamiento y/o las fases de } \\
\text { comercialización hacia los consumidores finales. La empresa } \\
\text { puede operar sola o en conjunto con otras empresas para } \\
\text { producir un bien o servicio. }\end{array}$ \\
\hline Porter, (2015) & Diferenciación & $\begin{array}{l}\text { Implica crear una propuesta de valor única e importante para } \\
\text { los compradores. La propuesta puede desarrollarse en: el } \\
\text { producto, medios de entrega, el servicio post-venta, entre } \\
\text { otros. }\end{array}$ \\
\hline $\begin{array}{l}\text { Sainz de Vicuña, } \\
\text { (2017) }\end{array}$ & Diversificación & $\begin{array}{l}\text { Se basa en el desarrollo de nuevos productos partiendo de su } \\
\text { producción principal, para ofrecerlos en los mercados } \\
\text { potenciales. La diversificación se centra en el producto o en el } \\
\text { mercado. }\end{array}$ \\
\hline
\end{tabular}

Fuente: Elaboración Propia.

En el nivel de estrategias de negocio, se pueden presentar combinaciones de estrategias, las cuales requieren de la definición de un plan a largo plazo que les permita a las empresas la consecución de sus objetivos, de estabilidad o crecimiento.

\section{La competitividad empresarial}

A pesar del tiempo que se lleva estudiando la competitividad, los autores tienen diferentes enfoques en función del momento en que han realizado sus propuestas, claramente influenciados por la situación del entorno. Esto ha dado paso a una multiplicidad de nociones que se complementan mayoritariamente. De acuerdo a Bernal, Fracica y Frost (2012), la competitividad de las empresas se refleja en la capacidad organizacional para poder producir bienes y/o servicios con dirección al mercado internacional, manteniendo o incrementando su rentabilidad.

Cabe destacar que la competitividad implica lograr mejores resultados para la empresa, involucrando a los procesos productivos, considerando sus capacidades para responder a los mercados internacionales que le permita sostenimiento y consolidación (Ortiz y Arredondo 2014).

Con respecto a los factores internos Ahumada y Perusquía (2016), han definido a la inteligencia estratégica como un elemento clave para la competitividad. Las empresas que formulan estrategias fundamentadas en conocimiento se consolidan, sobresalen y trascienden en el entorno empresarial. 
Dentro de este marco, la competitividad se relaciona estrechamente con la productividad debido a que los componentes inmersos en la estrategia competitiva están encaminados a desarrollar un producto de calidad. Efectivamente, las empresas pueden elaborar estrategias innovadoras, pero si el factor de calidad no está de por medio, está destinada al fracaso, como expresan Rojo, Bonilla y Masaquiza (2018).

Resulta enriquecedor identificar las diversas perspectivas que sobre competitividad exponen algunos autores. La diferencia de criterios nos lleva a corroborar que no hay una concepción exacta de lo que es competitividad. En la tabla 3 sintetizamos las perspectivas de varios autores.

Tabla 3.

Perspectivas sobre la competitividad.

\begin{tabular}{|c|c|}
\hline Autor & Descripción \\
\hline Licona y Turner (2014) & $\begin{array}{l}\text { La competitividad de una empresa se ve reflejada en la capacidad de cubrir } \\
\text { necesidades de un mercado, en dependencia de su nivel de productividad y } \\
\text { rentabilidad. }\end{array}$ \\
\hline Morales de Llano (2014) & $\begin{array}{l}\text { La competitividad está arraigada con el medio socioeconómico y ambiental en el } \\
\text { que se desenvuelve una empresa mediante un sistema de innovación y } \\
\text { mejoramiento continuo de la calidad, generando rentabilidad a largo plazo. }\end{array}$ \\
\hline $\begin{array}{c}\text { Horta, Silveira y Camacho } \\
\text { (2015) }\end{array}$ & $\begin{array}{l}\text { La capacidad de producir conocimiento por medio de la investigación y } \\
\text { desarrollo (I+D), y la inteligencia para ejecutarlo de manera eficiente con sus } \\
\text { recursos disponibles, son elementos claves para elevar el nivel de competitividad } \\
\text { de una empresa. }\end{array}$ \\
\hline $\begin{array}{l}\text { Rodríguez-Hernández, } \\
\text { Santoyo-Cortés Muñoz- } \\
\text { Rodríguez y Rodríguez- } \\
\quad \text { Padrón (2016) }\end{array}$ & $\begin{array}{l}\text { Los beneficios de una organización se logran a través de la propuesta, creación y } \\
\text { entrega de valor a sus socios, en un determinado tiempo, donde cada socio forma } \\
\text { parte de la actividad empresarial en la que se desenvuelve la organización para } \\
\text { alcanzar la competitividad. }\end{array}$ \\
\hline Silva y Guerrero (2017) & $\begin{array}{l}\text { El conjunto de elementos inmersos en la gestión del conocimiento permite a la } \\
\text { empresa crear estrategias adecuadas al tipo de negocio, promoviendo el } \\
\text { incremento del nivel de competitividad. Además, los componentes intangibles } \\
\text { como el capital humano y estructural representan activos determinantes para } \\
\text { obtener ventajas competitivas en el sector empresarial. }\end{array}$ \\
\hline Otero y Taddei (2018) & $\begin{array}{l}\text { La posición económica de un determinado sector promueve en las empresas su } \\
\text { fortalecimiento en el mercado local, e impulsa a que se extiendan hacia el } \\
\text { exterior. }\end{array}$ \\
\hline
\end{tabular}

Fuente: Elaboración Propia.

El análisis de la competitividad desde el enfoque de varios sectores refleja elementos que diferencian las prácticas organizacionales implementadas. En la tabla 4 se presenta un resumen de los resultados de las investigaciones de varios autores. 
Tabla 4.

Análisis de la competitividad desde diferentes sectores.

\begin{tabular}{|c|c|c|}
\hline Autor & Sector & Principales hallazgos \\
\hline $\begin{array}{l}\text { González, Carrión y } \\
\text { Palacios (2015) }\end{array}$ & Turístico & $\begin{array}{l}\text { Para asegurar el éxito en la organización es necesario } \\
\text { desarrollar un rol ético que permita modernizar el sistema } \\
\text { gerencial a través de su cultura y criterio de liderazgo, } \\
\text { identificando a la empresa como competitiva por sus } \\
\text { competencias personales. }\end{array}$ \\
\hline López-Torres et al. (2016) & $\begin{array}{l}\text { Manufacturero y de } \\
\text { servicios }\end{array}$ & $\begin{array}{l}\text { Existen diferentes formas de innovación, entre ellas se } \\
\text { destacan a la innovación de procesos, productos y } \\
\text { organizacional, cada una permite transformaciones } \\
\text { relevantes y eficientes en las empresas asegurando la } \\
\text { competitividad. }\end{array}$ \\
\hline $\begin{array}{l}\text { Fuentes, Osorio, y } \\
\text { Mungaray (2016) }\end{array}$ & Mipymes & $\begin{array}{l}\text { La base del crecimiento empresarial radica en el capital } \\
\text { humano. Su desarrollo personal y profesional es una } \\
\text { fortaleza, esto conduce a tener una ventaja competitiva por } \\
\text { su nivel de responsabilidad y cumplimiento con las metas } \\
\text { de la organización, frente a los demás competidores. }\end{array}$ \\
\hline $\begin{array}{c}\text { Guzmán, Tarapuez y Parra } \\
\text { (2017) }\end{array}$ & Servicios & $\begin{array}{l}\text { Para alcanzar ventajas competitivas y satisfacer las } \\
\text { necesidades de sus clientes, las organizaciones requieren } \\
\text { la implementación de estrategias de calidad mediante la } \\
\text { mejora continua, las cuales tienen un mayor impacto en el } \\
\text { desempeño de la organización. }\end{array}$ \\
\hline $\begin{array}{c}\text { Capa, Benítez y Capa } \\
\text { (2018) }\end{array}$ & Agrícola & $\begin{array}{l}\text { Para mantener un crecimiento constante desde el entorno } \\
\text { interno, es necesario que el líder fomente en sus } \\
\text { colaboradores el compromiso con la organización y el } \\
\text { cumplimiento de sus metas. Los resultados positivos de un } \\
\text { liderazgo efectivo, contribuyen a diseñar y ejecutar } \\
\text { estrategias que conlleven a obtener ventajas competitivas. }\end{array}$ \\
\hline
\end{tabular}

Fuente: Elaboración Propia.

En las líneas superiores hemos identificado los resultados de varios estudios respecto a la competitividad. Los autores coinciden en que las variables más importantes para generar competitividad son: la cultura organizacional, el capital humano, el liderazgo, la innovación, la implementación de estrategias de calidad y la gestión del conocimiento.

Las estrategias definidas para generar ventajas competitivas se constituyen en elementos determinantes en el crecimiento de una organización. Los líderes analizan acciones que les permita conseguir características diferenciadoras, analizando las fortalezas y debilidades internas y estimando los riesgos y oportunidades del entorno, a esto se denomina desarrollo de estrategias competitivas.

\section{Evolución del sector camaronero}

En la década de los 90, el sector camaronero ecuatoriano enfrentaba una dura crisis sanitaria y económica a causa del virus de la mancha blanca, que provocó la reducción de la producción y la inactividad de varias hectáreas. Para la producción se utilizaba la larva silvestre, aún no se producían larvas en los laboratorios y el sistema de cultivo era intensivo.

La recuperación del sector camaronero fue impulsada por transformaciones que abarcan toda la cadena productiva. Así, con el objetivo de superar los problemas críticos de la producción 
se utilizaron larvas mejoradas y de mayor resistencia a las enfermedades. Los cambios en los sistemas de cultivo, alimentación, e infraestructura condujeron al fortalecimiento y crecimiento del sector, las hectáreas dedicadas a la producción de camarón se duplicaron aumentando la producción (Revista Líderes, 2017).

Demostrar la sanidad del camarón es importante, es un requisito para el ingreso al mercado exterior. Las empresas ecuatorianas han adoptado sistemas de aseguramiento de la calidad para robustecer el prestigio del producto. En el año 2018, por iniciativa de la Cámara Nacional de Acuacultura (en adelante CNA) se lanzó la certificación Sustainable Shrimp Partnership (SSP), concebido como un programa para garantizar que el camarón se cultiva sin el uso de antibióticos, reduciendo los impactos negativos al medio ambiente. Esta iniciativa representa el factor diferenciador en la promoción del camarón en las ferias nacionales y las ruedas de negocio en cooperación con ProEcuador (Aquacultura, 2018).

Después de varios años de mejoras en el sector, se incrementaron las ventas al exterior y actualmente el camarón es uno de los principales productos de exportación no petrolera. Los principales destinos de exportación son: Vietnam, Estados Unidos, España, Francia, Italia y China (ProEcuador, 2017).

\section{Metodología}

Esta investigación se enmarca en un enfoque cualitativo, de carácter descriptivo y exploratorio. La evidencia empírica se refleja en la información secundaria de las webs empresariales y publicaciones sobre su situación. Se aplica un muestreo no aleatorio. En el contexto de investigación que nos ocupa, el alcance del estudio se asocia a la identificación de las estrategias adoptadas por las empresas ecuatorianas exportadoras de camarón que reflejan los mejores resultados económicos del sector.

Los estudios de Santos-Álvarez y García-Merino (2018) establecen un vínculo entre la estrategia y el estudio de casos, porque su objetivo es dar a conocer su relación con todo el ámbito empresarial. En este orden de ideas, consideramos que la metodología del estudio de casos es adecuada para los objetivos de investigación planteados. A continuación, recogemos las precisiones teóricas de algunos autores, las cuales nos permiten respaldar la elección de la metodología:

Siguiendo a Yin (1989), el estudio de casos analiza un fenómeno objeto de estudio y satisface las necesidades del conocimiento en un ambiente concreto que proporciona confianza al investigador, valiéndose de múltiples fuentes secundarias que abundan en información propia de una empresa. Yin (2001) propone que, el método estudio de casos tiene 6 formas distintas para obtener evidencias: documentos, registros en archivos, entrevistas, observación directa, observación participante y artefactos físicos.

Eisenhardt (1989), señala que en los estudios de casos múltiples es necesario tomar de 4 a 10 casos para obtener veracidad en los resultados, de manera que, si un investigador utiliza un solo caso de estudio, este necesitaría de fórmulas estadísticas para refutar su veracidad, por consiguiente, Strauss y Corbin (2002), manifiestan que la investigación cualitativa, genera 
grandes descubrimientos sin la ejecución de técnicas estadísticas, simplemente, se enfoca en la actividad diaria del objeto de estudio.

Villarreal (2017), recomienda para el estudio de casos la aplicación de los recursos a partir de evidencia documentada que proviene de entrevistas y la observación directa. Al hilo de estas ideas, Nájera y Montenegro (2017), concuerdan en que las investigaciones de tipo cualitativas requieren de un análisis exhaustivo de diferentes fuentes secundarias.

Para relacionar en profundidad la metodología estudio de caso con la presente investigación, planteamos los siguientes ejemplos: Graterol-López y Sigala-Paparella (2014) realizaron estudios de casos a 6 empresas de producción y servicios, recabando información mediante trabajos de campo y casos documentales. Peña y Ari (2015) hicieron un estudio de casos en una empresa cementera aplicando entrevistas con preguntas estructuradas, mientras tanto, Trillo y Hernández (2016) aplican la metodología estudio de casos en las empresas exportadoras y productoras de camarón utilizando el análisis documental, análisis sectorial, entrevistas semiestructuradas y observación directa.

Al hilo de los argumentos descritos que han servido de base, para la presente investigación se aplicó la observación directa, el análisis documental de investigaciones existentes e información descrita por las empresas en sus páginas webs. El análisis de los recursos de información nos permitió describir la situación de las unidades de estudio. La base teórica sobre los tipos de estrategias se empleó para contrastar con las acciones más visibles que las empresas han ejecutado hasta el momento. Esta asociación nos llevó a conectar la teoría con la práctica de las empresas, obteniendo finalmente la caracterización de los tipos de estrategias en el sector exportador de camarón en Ecuador.

Para la selección de la muestra de empresas camaroneras recurrimos al ranking de las empresas ecuatorianas del top 100 realizado por (Revista Ekos, 2018), el mismo que establece el volumen de ventas como variable para la clasificación. El estudio de casos comprende a las empresas: Industrial Pesquera Santa Priscila (en adelante Caso 1), Expalsa (en adelante Caso 2), Omarsa (en adelante Caso 3), Songa (en adelante Caso 4), Promaoro (en adelante Caso 5).

A continuación, se presentan la evidencia empírica a través de los cinco casos objeto de estudio, desde los cuales pretendemos abordar el objeto de estudio.

\section{Caso 1}

Santa Priscila inicia sus actividades de producción y comercialización de camarón en el año 1973. En 1999 empezó a diversificar sus productos a través de la tilapia y la exportación de frutas frescas como el mango.

El camarón de Santa Priscila se siembra en baja viscosidad. La empresa se mantiene en constante innovación a través de prácticas sostenibles aplicadas en los procesos de bioseguridad. Se caracteriza principalmente por su productividad y compromiso ambiental, es una de las primeras empresas en el Ecuador del sector acuícola en recibir la certificación Punto Verde. Posee la certificación ETI "Iniciativa de Comercio Ético", mediante la cual se garantiza a los 
consumidores que los productos se obtienen y procesan cumpliendo normas éticas. Asimismo, el camarón ecuatoriano también se volvió atractivo y estableció una alianza para una organización líder en temas ambientales (Seafood Watch), para promocionar productos de Santa Priscila.

Santa Priscila es proveedora autorizada de una de las más importantes cadenas de supermercados en productos orgánicos de Estados Unidos (Whole Foods Market). No obstante, las ventas se concentran en el mercado chino, en este sentido, las alianzas que el gobierno ecuatoriano ha realizado han sido un gran impulso para este mercado.

En el 2017, las fincas camaroneras de Santa Priscila se insertaron al Plan de Electrificación que lideró el Ministerio de Acuacultura y Pesca y la CNA en conjunto con el Gobierno Nacional. El Plan de Electrificación tiene por objetivo reemplazar el uso del diésel por la energía eléctrica renovable en todos sus sistemas productivos, aplicable en la fase de aireación eléctrica, succión y alimentación mecánica en las fincas camaroneras. La empresa mantiene una participación activa con las instancias de gobierno que promueven el fortalecimiento de los sectores productivos y el aprovechamiento de oportunidades comerciales.

\section{Caso 2}

Expalsa es una empresa líder en el mercado que se destaca por vocación ambiental, trazabilidad y sustentabilidad desde 1984. En el 2017 Expalsa fue la segunda empresa camaronera en liderar el mercado con una utilidad de 6,43\%. Se caracteriza por su producción orgánica y certificaciones, con las cuales se respalda que sus productos no utilizan antibióticos y garantizan el cumplimiento del sistema de calidad al mercado internacional.

\section{Caso 3}

Omarsa inició el negocio de camarón en el año de 1977. Es una de las primeras empresas a nivel mundial que se benefició de la certificación ASC. Actualmente el 100\% de sus hectáreas son de producción orgánica y cuenta con un Plan de Responsabilidad Social y Corporativa (RSE) que tiene como base: el medio ambiente, la comunidad y los trabajadores. Omarsa se destaca por operar bajo una estricta política de control de calidad y principios de preservación ambiental, con el fin de proveer un producto de calidad y asegurar la sustentabilidad a largo plazo. En el año 2014 la empresa ganó un reconocimiento por los altos estándares de calidad de su producto. El recurso humano es considerado como pieza clave en el desarrollo empresarial, por lo cual, esta empresa implementa programas de capacitación de manera constante, cubriendo todas las áreas administrativas y operativas. En el año 2013 Omarsa obtuvo un reconocimiento nacional por diversificación de mercados no tradicionales.

\section{Caso 4}

Con más de tres décadas de operaciones, la Sociedad Nacional de Galápagos (en adelante Songa) se ha convertido en una de las principales exportadoras de camarón a nivel nacional. Sus procesos de producción cumplen con los estándares de calidad que el mercado exige. Se distingue de otras empresas por la aplicación de prácticas de acuicultura responsable, la certificación de Buenas Prácticas de Acuicultura (BAP) y su compromiso de responsabilidad 
social y ambiental. Para prevenir la contaminación, la empresa aplica programas de tratamiento del agua con tecnología moderna que asegura la no contaminación y el cumplimiento de las leyes ambientales del país.

Las instalaciones de las camaroneras proveedoras de Songa, disponen de tecnología de punta y personal altamente capacitado. También, ha ganado reconocimiento a nivel nacional e internacional por su calidad y consistencia. Entre sus mercados se identifica a China Continental, Corea del Sur y Estados Unidos.

\section{Caso 5}

Promaoro se encuentra ubicada en la provincia de El Oro. Ha ganado reconocimiento desde el año 2012 como productora, procesadora, comercializadora y exportadora de camarón de excelente calidad. La empresa se enfoca en la trazabilidad total, respeto al medio ambiente y social, de manera que, su principal compromiso es la calidad de su producto.

Como principales clientes de Promaoro se destacan los mercados de Europa, Asia y Estados Unidos. Conforme a su trayectoria, esta empresa también ha entablado alianzas estratégicas con el gobierno nacional, ha sido beneficiaria de créditos enmarcados en el cambio de la matriz productiva e innovación. En el marco del Plan de Electrificación ha reemplazado sus equipos de producción con el objetivo de reducir costos y contribuir a una producción más limpia y responsable.

\section{Resultados}

Para la presentación de los resultados se han sistematizado los hallazgos que identificamos como estrategias. Partiendo de una caracterización, describimos: estrategia de crecimiento (estrategia de integración vertical y estrategia de diversificación) y estrategias competitivas-diferenciación (certificaciones como estrategias de diferenciación). Habiendo seleccionado a las empresas de mayor desempeño en el sector, las acciones que reseñamos se reflejan como estrategias que les han permitido mantenerse competitivas.

La estrategia de integración vertical en los casos objeto de estudio se materializa desde la inversión en infraestructura y equipos. El negocio principal de las exportadoras de camarón, esto es, la comercialización de camarón en los mercados internacionales, se ha expandido hacia otras actividades de la cadena de valor. Esto les ha permitido a las empresas abaratar costos, asegurar la calidad desde el abastecimiento de la materia prima, controlar el procesamiento del balanceado principal alimento del camarón. En la tabla 5 se recogen características de las empresas objeto de estudio, las cuales asocian a la estrategia de integración vertical. 
Tabla 5.

Estrategia de Integración Vertical.

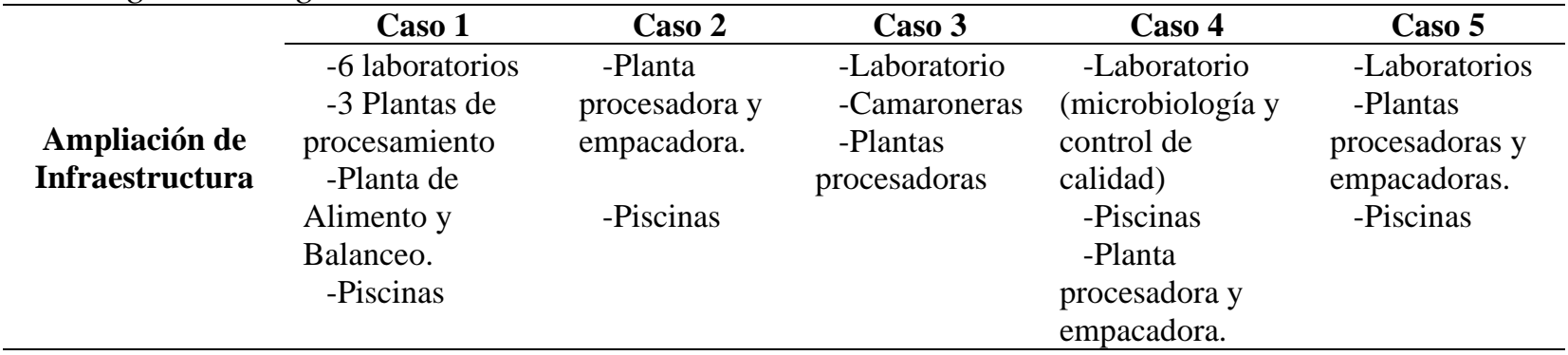

Fuente: Elaboración Propia

Entre los principales cambios que han experimentado los mercados de bienes alimenticios se destaca una mayor conciencia de los consumidores sobre el origen y forma en que se obtienen los productos. Los consumidores que eligen sus alimentos a partir de la información asociada a la forma de producción, se han convertido en un segmento de crecimiento sostenido. Las empresas exportadoras de camarón que han realizado una lectura de las nuevas exigencias del mercado, se caracterizan por adoptar acciones proactivas hacia el medio ambiente, junto a políticas y estándares de calidad que marcan las diferencias entre sus competidores.

A continuación, en la tabla 6 se describen las estrategias de diferenciación a partir de la sustentabilidad, compromiso social y calidad-trazabilidad.

Tabla 6.

Estrategia de Diferenciación

\begin{tabular}{|c|c|c|c|c|c|}
\hline Valor agregado & Caso 1 & Caso 2 & Caso 3 & Caso 4 & Caso 5 \\
\hline Sustentabilidad & $\begin{array}{l}\text {-Producen en sus } \\
\text { propias plantas. } \\
\text {-Personal idóneo y } \\
\text { certificaciones. } \\
\text {-Buscan la mejora } \\
\text { continua } \\
\text { erradicando el } \\
\text { impacto ambiental y } \\
\text { posee certificación } \\
\text { punto verde. }\end{array}$ & $\begin{array}{l}\text {-Buenas } \\
\text { prácticas de } \\
\text { acuicultura } \\
\text { bajo estrictas } \\
\text { certificaciones } \\
\text {-Producción } \\
\text { orgánica y } \\
\text { productos sin } \\
\text { antibióticos. }\end{array}$ & $\begin{array}{l}\quad \text {-Bienestar } \\
\text { animal, no } \\
\text { utiliza } \\
\text { materiales } \\
\text { tóxicos. }\end{array}$ & $\begin{array}{l}\text {-Certificación } \\
\text { ASC y personal } \\
\text { calificado. } \\
\text {-Comprometida } \\
\text { en } \\
\text { responsabilidad } \\
\text { social y } \\
\text { ambiental. }\end{array}$ & $\begin{array}{l}\text {-Producción } \\
\text { orgánica, } \\
\text { respetando el } \\
\text { medio } \\
\text { ambiente. }\end{array}$ \\
\hline $\begin{array}{l}\text { Compromiso - } \\
\text { Social }\end{array}$ & $\begin{array}{l}\text {-Programas para } \\
\text { erradicar el trabajo } \\
\text { infantil. } \\
\text {-Atención } \\
\text { prioritaria en } \\
\text { Tugaduaja y } \\
\text { Engungacon. } \\
\text { - } \\
\text { Acondicionamiento } \\
\text { de áreas verdes, etc. }\end{array}$ & $\begin{array}{l}\text {-Proyecto } \\
\text { Nuestros } \\
\text { Hijos. } \\
\text {-Camaronera } \\
\text { Comunitaria } \\
\text { Engunga. }\end{array}$ & $\begin{array}{l}\text {-Plan de } \\
\text { Responsabilidad } \\
\text { Social y } \\
\text { Corporativo. } \\
\text {-Conservación } \\
\text { del Manglar. } \\
\text {-Agua para la } \\
\text { Comunidad }\end{array}$ & $\begin{array}{l}\text {-Proyectos para } \\
\text { erradicar trabajo } \\
\text { infantil, } \\
\text { seguridad para } \\
\text { sus empleados, } \\
\text { etc. }\end{array}$ & $\begin{array}{l}\quad \text {-Asegura a } \\
\text { sus } \\
\text { colaboradores } \\
\text { y presta } \\
\text { atención } \\
\text { prioritaria a } \\
\text { zonas } \\
\text { aledañas. }\end{array}$ \\
\hline $\begin{array}{l}\text { Calidad - } \\
\text { Trazabilidad }\end{array}$ & $\begin{array}{ll}- & \text { Recepción y } \\
- & \text { Pesaje, lava } \\
- & \text { Empaque, p } \\
- & \text { Congelaciór } \\
- & \text { Embarque. }\end{array}$ & $\begin{array}{l}\text { dificación del } \\
\text { selección, cla } \\
\text { je y rotulación } \\
\text { ncartonado y }\end{array}$ & $\begin{array}{l}\text { narón. } \\
\text { cación. } \\
\text { acenamiento. }\end{array}$ & & \\
\hline
\end{tabular}


Fuente: Elaboración Propia.

Para Zarta (2018), la sustentabilidad involucra a la producción limpia en los sistemas de la acuicultura, fundamentándose en la contaminación ambiental y en los recursos naturales que se agotan día a día. Para tal efecto, las empresas camaroneras en conjunto con la CNA han optado por recuperar el manglar de la costa ecuatoriana, reforestando más de 2200 hectáreas hasta el presente.

Sin duda la preservación del medio ambiente es uno de los compromisos sociales y ambientales que las empresas camaroneras han adaptado en sus prácticas de manufactura, por lo cual, es importante recalcar que la sostenibilidad se traza en un cambio de conciencia, respetando los derechos, obligaciones y condiciones medioambientales tal como lo menciona López (2015).

La trazabilidad, siguiendo a Herrera y Orjuela (2014), implica que todos los procesos de la cadena productiva del sector camaronero deben tener un respectivo control y seguimiento. Para esto se codifican cada uno de sus procesos hasta llegar al consumidor final, lo cual abarca desde la selección de la larva hasta el embarque del producto. Las empresas de nuestro estudio cumplen con la trazabilidad como parte de los mecanismos para asegurar la información de los procesos y su calidad.

Por otra parte, las exigencias de los mercados dinámicos plantean a las empresas la necesidad de aplicar buenas prácticas y mantener la calidad en sus procesos y productos. La estrategia de las empresas en este ámbito se refleja en la innovación de procesos, sistemas de inocuidad, acciones que finalmente se concretan en certificaciones internacionales de prestigio en los mercados.

En la tabla 7, se resumen las principales certificaciones de calidad que han conseguido las empresas camaroneras objeto de estudio, las cuales caracterizamos como estrategia de diferenciación. 
Tabla 7.

Certificaciones como estrategia de diferenciación.

\begin{tabular}{|c|c|c|c|c|c|c|}
\hline \multicolumn{7}{|c|}{ Certificaciones de Calidad } \\
\hline Certificaciones & Descripción & $\begin{array}{l}\text { Cas } \\
\text { o } 1\end{array}$ & $\begin{array}{l}\text { Cas } \\
\text { o } 2\end{array}$ & $\begin{array}{l}\text { Cas } \\
\text { o } 3\end{array}$ & $\begin{array}{l}\text { Cas } \\
\text { o } 4\end{array}$ & $\begin{array}{l}\text { Cas } \\
\text { o } 5\end{array}$ \\
\hline $\begin{array}{r}\text { ASC / Aquaculture } \\
\text { Stewardship Council }\end{array}$ & $\begin{array}{l}\text { Consejo de Administración } \\
\text { de Acuicultura. }\end{array}$ & $\mathrm{x}$ & & $\mathrm{x}$ & $\mathrm{x}$ & \\
\hline Naturland & $\begin{array}{l}\text { Asociación de Productores } \\
\text { Orgánicos. }\end{array}$ & & $\mathrm{x}$ & $\mathrm{x}$ & & \\
\hline BAP & $\begin{array}{l}\text { Buenas Prácticas de } \\
\text { Acuicultura. }\end{array}$ & & & $\mathrm{x}$ & $\mathrm{x}$ & \\
\hline GLOBAL G.A.P & Buenas Prácticas Agrícolas. & & $\mathrm{x}$ & $\mathrm{x}$ & & \\
\hline BRC & Seguridad Alimentaria. & $\mathrm{x}$ & $\mathrm{x}$ & $\mathrm{x}$ & $\mathrm{x}$ & $\mathrm{X}$ \\
\hline НАССР & $\begin{array}{l}\text { Análisis de Peligros y Puntos } \\
\text { Críticos de Control. }\end{array}$ & $\mathrm{x}$ & $\mathrm{x}$ & & $\mathrm{x}$ & \\
\hline Punto Verde & $\begin{array}{l}\text { Reconocimiento Ambiental } \\
\text { Ecuatoriano. }\end{array}$ & $\mathrm{x}$ & & & & \\
\hline AB (Agriculture Biologique) & Producciones Orgánicas. & & $\mathrm{x}$ & & & \\
\hline Basc & $\begin{array}{l}\text { Alianza Empresarial para el } \\
\text { Comercio Justo. }\end{array}$ & & $\mathrm{x}$ & $\mathrm{x}$ & $\mathrm{x}$ & $\mathrm{x}$ \\
\hline Sedex & $\begin{array}{l}\text { Organización Global de } \\
\text { Comercio Ético. }\end{array}$ & & $\mathrm{x}$ & $\mathrm{x}$ & & \\
\hline
\end{tabular}

Fuente: Elaboración Propia a partir de ProEcuador ( 2017).

Panorama Acuícola (2019) indica que las certificaciones representan un aval de cumplimiento hacia el cuidado del medio ambiente, seguridad alimentaria, bienestar animal y seguridad laboral. Las empresas pueden diferenciarse por la capacidad de demostrar confianza a los consumidores, en procesos y productos. Finalmente, las certificaciones añaden valor a las empresas y favorece la entrada y permanencia en los mercados internacionales.

Con la información recopilada de las empresas objeto de estudio, la estrategia de diversificación se relaciona con la existencia de marcas y productos dirigida a los mercados internacionales. En la tabla 8 se describen los hallazgos asociados a este tipo de estrategia. 
Tabla 8.

Estrategia de Diversificación.

\begin{tabular}{|c|c|c|}
\hline Empresas & Marcas & Productos \\
\hline Caso 1 & $\begin{array}{l}\text { Golden Sea Shrimp } \\
\text { IQF Camarón Precocido Shell on IQF } \\
\text { Golden con Cáscara } \\
\text { IQF PYD Golden Grande Filete de } \\
\text { Tilapia 2-3 Rojito } \\
\text { Naturalia Organic Tilapia }\end{array}$ & $\begin{array}{l}\text { Camarón congelado en bloque o IQF en salmuera. } \\
\text { Tilapia } \\
\text { Piel y Escamas de tilapia roja. } \\
\text { Mango }\end{array}$ \\
\hline Caso 2 & $\begin{array}{l}\text { Head Less } \\
\text { Head On }\end{array}$ & $\begin{array}{l}\text { Camarón orgánico Head-on } \\
\text { Head-lees }\end{array}$ \\
\hline Caso 3 & $\begin{array}{l}\text { Vanoni's } \\
\text { Vanoni's Organic } \\
\text { Vanoni's Langua }\end{array}$ & $\begin{array}{l}\text { Camarón entero con cola, valor agregado. } \\
\text { Tití. }\end{array}$ \\
\hline Caso 4 & $\begin{array}{l}\text { Number One } \\
\text { Marcol Champmar } \\
\text { Ecuagold }\end{array}$ & $\begin{array}{l}\text { Camarones crudos y cocidos con piel, cola pelada } \\
\text { (crudos y cocidos) }\end{array}$ \\
\hline Caso 5 & $\begin{array}{l}\text { Ocean Deli } \\
\text { Santa Rosa } \\
\text { Puerto Jelí } \\
\text { PFS }\end{array}$ & Camarón con cola y camarón entero. \\
\hline
\end{tabular}

Fuente: Elaboración Propia.

Cabe destacar que las alianzas entre el sector camaronero y las instituciones de gobierno, han robustecido la imagen del camarón ecuatoriano en los mercados internacionales. Así, la creación de la marca sectorial ha potenciado la diferenciación de la competencia, y el posicionamiento en los mercados como un producto de calidad (Ministerio de Comercio Exterior, 2017).

\section{Conclusiones}

El dinamismo de los mercados ha llevado a las empresas del sector camaronero a la combinación de estrategias para ser competitivas. Entre la evidencia empírica de las empresas se destacan prácticas organizacionales asociadas con las estrategias de crecimiento. Así, la integración vertical se ve reflejada en las inversiones realizadas para expandir las actividades de la cadena de valor, superando la comercialización. Dentro de la estrategia de crecimiento se reconoce la estrategia de diversificación, la cual se manifiesta a través de los productos y marcas creadas para los mercados internacionales.

Las estrategias competitivas están presentes en la diferenciación conseguida por las empresas a través de las certificaciones de programas gestión de la calidad, las buenas prácticas de manufactura, sustentabilidad y responsabilidad social. Este tipo de estrategias, permite que el producto camarón cumpla con las normativas y estándares de calidad que exigen los mercados internacionales, y a su vez genera confianza a los consumidores.

Por otro lado, las alianzas con instituciones gubernamentales se han convertido en fuentes de oportunidades para la renovación tecnológica, la incursión en nuevos mercados y el posicionamiento del camarón ecuatoriano a través de la marca país. 
Finalmente, desde esta investigación exploratoria se contribuye a identificar las estrategias de las empresas camaroneras a través de evidencia empírica derivada de datos secundarios. Por otro lado, reconocemos que la metodología de estudio de casos no nos permite generalizar los hallazgos. Dicha limitación a su vez nos proporciona la pauta para futuras investigaciones, desde las cuales se debería considerar trabajar con muestras paramétricas y la obtención de datos primarios que posibiliten el análisis cuantitativo de las estrategias y sus relaciones.

\section{Bibliografía}

Ahumada Tello, E., \& Perusquia Velasco, J. M. (2016). Inteligencia de negocios: estrategia para el desarrollo de competitividad en empresas de base tecnológica. Contaduría y Administración, 61(1), 127-158.

Andrews, K. R. (1971). The concept of corporate strategy. Homewood, III., Dow Jones-Irwin.

Ansoff, I. (1998). La Dirección estratégica en la práctica empresarial. México D.F: Pearson Education.

Aquacultura. (Abril de 2018). Ecuador presenta "Sustainable Shrimp Partnership (SSP)". (122), $1-80$

Baena Graciá, V. (2011). Fundamentos de marketing: entorno, consumidor, estrategia e investigación comercial. Barcelona: UOC.

Banco Central del Ecuador. (2015). Exportaciones por actividad económica. Obtenido de https://www.bce.fin.ec

Bernal Torres, C. A., Fracica Naranjo, G., \& Frost González, J. S. (2012). Análisis de la relación entre la innovación y la gestión del conocimiento empresarial en una muestra de empresas en la ciudad de Bogotá. Estudios Gerenciales, 28, 303-315.

Capa Benítez, L. B., Benítez Narváez, R. M., \& Capa Benítez, X. R. (2018). El liderazgo como fuente de ventaja competitiva para las organizaciones. Universidad y Sociedad, 10(2), 273-284.

Chandler, A. D. (1962). Strategy and Structure: Chapters in the History of American Enterprise. Boston: MIT Press.

Eisenhardt, K. M. (1989). Building Theories from Case Study Research. The Academy of Management Review, 14(4), 532-550.

Fuentes, N., Osorio, G., \& Mungaray, A. (2016). Capacidades intangibles para la competitividad microempresarial en México. Revista Problemas del desarrollo, 186(47), 83-106.

Gallo Mendoza, J. G. (2017). La orientación al mercado, el dinamismo del entorno y la tipología estratégica de la pequeña y mediana industria: un análisis mediante modelo de ecuaciones estructurales. INNOVA Research Journal, 2(8.1), 64-85.

González Rosas, E. L., Carrión García, A., \& Palacios Marqués, D. (2015). El liderazgo por competencias y el EFQM. Investigación Administrativa, 44(116), 10-24.

Graterol-López, H., \& Sigala-Paparella, L. E. (2014). Empresas de internacionalización acelerada: estudio de casos en Venezuela. Estudios Gerenciales, 30(133), 461-468.

Guzmán Díaz, B. E., Tarapuez, E., \& Parra Hernández, R. (2017). Estrategia y calidad en las empresas colombianas de servicios. Revista Venezolana de Gerencia, 22(80), 593-609.

Herrera Ramírez, M. M., \& Orjuela Castro, J. A. (2014). Perspectivas de trazabilidad en la cadena de suministros de frutas: un enfoque desde la dinámica de sistemas. Ingeniería, 19(2), 63-84. 
Horta, R., Silveira, L., \& Camacho, M. (2015). Competitividad e innovación en la industria manufacturera en el Uruguay. Revista Iberoamericana de ciencia y tecnología y sociedad, 10(28), 23-49.

Jones, G. R., \& George, J. M. (2006). Administración contemporánea. México D.F.: The McGraw-Hill.

Licona Michel, Á., \& Turner Barragán, E. H. (2014). Competitividad sistémica y pilares de la competitividad de Corea del Sur. Análisis Económico, 29(72), 155-175.

López Pardo, I. (2015). Sobre el desarrollo sostenible y la sostenibilidad: conceptualización y crítica. BARATARIA. Revista Castellano-Manchega de las Ciencias Sociales, 20, 111128.

López-Torres, G. C., Maldonado Guzmán, G., Pinzón Castro, Y., \& García Ramírez, R. (2016). Colaboración y actividades de innovación en Pymes. Contaduría y Administración, 61(3), 568-581.

Luna González, A. C. (2014). Administración estratégica. México D.F.: Patria.

Manfredi, L. C., \& González Aragón, É. (2017). Autopartes del Caribe. Negociando estratégicamente. Estudios Gerenciales, 33(144), 309-316.

Ministerio de Comercio Exterior. (2017). Camarón Ecuatoriano contará con marca sectorial. Obtenido de https://www.comercioexterior.gob.ec/camaron-ecuatoriano-contara-conmarca-sectorial/

Morales de Llano, E. (2014). La dimensión territorial de la competitividad. Economía y Desarrollo, 151(1), 71-84.

Mora-Riapira, E. H., Vera-Colina, M. A., \& Melgarejo-Molina, Z. A. (2015). Planificación estratégica y niveles de competitividad de las Mipymes del sector comercio en Bogotá. Estudios Gerenciales, 31(134), 79-87.

Nájera, S., \& Montenegro Moreno, D. H. (2017). La generación e implementación de la estrategia en una empresa y su influencia en las políticas de calidad en la industria Ecuatoriana: un estudio de caso. INNOVA Research Journal, 2(8), 136-140.

Ohmae, K. (1989). La mente estratega. Madrid: McGraw Hill.

Ortiz Paniagua, C. F., \& Arredondo Ortega, E. (2014). Competitividad y factores de éxito de empresas desarrolladoras de software. Revista Venezolana de la Información, Tecnología y Conocimiento, 11(3), 49-73.

Otero Flores, S., \& Taddei Bringas, C. (2018). Competitividad de empresas familiares. Interciencia, 43(4), 236-241.

Panorama Acuícola. (2019). Hablando de certificaciones por Peter A. Cook. Panorama Acuícola, 24(3), 1-116.

Peña Romero, J. P., \& Ari Zilber, M. (2015). Innovación en el sector cementero de Colombia: estudio de caso Cementos Tequendama. Estudios Gerenciales, 31(135), 171-182.

Porter, M. E. (2015). Estrategia competitiva: técnicas para el análisis de los sectores industriales y de la competencia (2da ed.). México: Patria.

ProEcuador. (2017). Camarón. Obtenido de https://www.proecuador.gob.ec/ficha-de-camaron/

Revista Ekos. (2018). Ekos. Negocios Sostenibles. 294. Obtenido de http://revista.ekosnegocios.com

Revista Líderes. (2017). El camarón ecuatoriano tiene un plan de posicionamiento global. Obtenido de https://www.revistalideres.ec/lideres/camaron-ecuatoriano-planposicionamiento-global.html 
Revista Líderes. (2017). La industria del sector camaronero reflotó con fuerza. Obtenido de https://www.revistalideres.ec/lideres/industria-nacional-camaron-refloto-fuerza.html

Rodríguez-Hernández, O., Santoyo-Cortés, V. H., Muñoz-Rodríguez, M., \& Rodríguez-Padrón, B. (2016). La posición competitiva de las organizaciones cafetaleras en Huatusco,Veracruz. Revista Mexicana de Ciencias Agrícolas(15), 2965-2979.

Rojo, M. A., Bonilla, D. M., \& Masaquiza, C. S. (2018). El desarrollo de nuevos productos y su impacto en la producción: caso de estudio BH Consultores. Universidad y Sociedad, 10(1), 134-142.

Sainz de Vicuña Ancín, J. M. (2017). El plan estratégico en la práctica. Madrid: ESIC.

Santos-Álvarez, M. V., \& García-Merino, T. (2018). Los experimentos en la investigación de estrategia: una mirada múltiple. Estudios Gerenciales, 34(147), 229-237.

Silva Siu, D. R., \& Guerrero Bejarano, M. A. (2017). La gestión del conocimiento y los sistemas de información como fuentes de ventaja competitiva para las empresas. INNOVA Research Journal, 2(4), 73-76.

Strauss, A., \& Corbin, J. (2002). Bases de la investigación cualitativa. Técnicas y procedimientos para desarrollar la teoría fundamentada. Medellín: Universidad de Antioquia.

Tarapuez, E., Guzmán, B., \& Parra Hernández, R. (2016). Estrategia e innovación en las Mipymes colombianas ganadoras del premio Innova 2010-2013. Estudios Gerenciales, 32(139), 170-180.

Thompson, A. A. (1994). Dirección y administración estratégica: conceptos, casos y lecturas. México: Irwin.

Thompson, A. A., Gamble, J. E., Peteraf, M. A., \& Strickland III, A. J. (2015). Administración Estratégica. Teoría y Casos. México D.F.: McGraw-Hill Interamericana.

Trillo Holgado, M. A., \& Hernández Escobar, J. A. (2016). La dinámica empresarial basada en los intangibles un estudio de caso sobre las empresas camaroneras venezolanas. Economía Industrial(399), 143-152.

Villarreal Larrinaga, O. (2017). Is it desirable, necessary and possible to perform research using case studies? Cuadernos de Gestión, 17(1), 147-172.

Yin, R. (2001). Estudo de caso. Planejamento e Métodos. Porto Alegre: Bookman.

Yin, R. K. (1989). Case Study Research. Design and Methods, Applied Social Research Methods Series (Vol. 5). London: Sage Publications.

Zarta Ávila, P. (2018). La sustentabilidad o sostenibilidad: un concepto poderoso para la humanidad. Tabula Rasa(28), 409-423. 